\title{
Magnitude of Bite Force that is Interpreted as Clenching in Patients with Temporomandibular Disorders: A Pilot Study
}

\section{Nishiyama A*, Otomo N, Tsukagoshi K, Tobe S and Kino K}

Section of Temporomandibular Joint and Oral Function, Department of Comprehensive Patient Care, Graduate School, Tokyo Medical and Dental University, Tokyo, Japan

*Corresponding author: Akira Nishiyama, Department of Comprehensive Patient Care, Graduate School, Tokyo Medical and Dental University, 1-5-45, Yushima, Bunkyo-ku, Tokyo 113-8549, Japan, Tel: +81-3-5803-5713; Fax: +81-3-5803-5713; E-mail: anishi.tmj@tmd.ac.jp

Rec date: Jun 19, 2014, Acc date: Aug 11, 2014, Pub date: Aug 13, 2014

Copyright: (c) 2014 Nishiyama A, et al. This is an open-access article distributed under the terms of the Creative Commons Attribution License, which permits unrestricted use, distribution, and reproduction in any medium, provided the original author and source are credited

\section{Abstract}

Introduction: The magnitude of bite force that is interpreted as clenching (imagined clenching level: ICL) in patients with temporomandibular disorders (TMD) remains unknown. We aimed to investigate the relationship between ICL and symptoms of TMD to evaluate the suitability of the term clenching for interpreting bite force in these patients.

Methods: A total 76 patients (16 men, 60 women; age, $41.1 \pm 16.5$ years) with temporomandibular disorders treated at the Temporomandibular Joint Clinic at Tokyo Medical and Dental University were selected. All patients completed a questionnaire that evaluated gender, age, pain intensity at rest and during maximum mouth opening and chewing, ICL, and TMD-related limitation of daily functions. Pain intensity and ICL were estimated using a visual analog scale for pain. TMD-related limitation of daily functions was evaluated using a 10-item questionnaire.

Results: The mean (SD) visual analog scale score for ICL was $77.4( \pm 18.7 \mathrm{~mm}$ ) and was significantly correlated with the pain intensity during maximum mouth opening and chewing, and the TMD-related limitation of daily function score; however, only the latter was identified as significant in the multiple regression analysis $(P<0.05)$.

Conclusions: TMD patients imagined the clenching force at a magnitude of approximately $70 \%$ of the maximum bite force. Patients are considered unable to sustain clenching at this magnitude; therefore, clenching is not a preferred term when administering counseling and behavioral therapy to TMD patients.

Keywords: Imagined clenching level; Temporomandibular disorders; Questionnaire; Visual analog scale

\section{Abbreviations:}

ICL: Imagined clenching level; LDF-TMDQ: TMD-related limitation of daily functions; RDC/TMD: Research Diagnostic Criteria for Temporomandibular Disorders; TMD: Temporomandibular disorders; VAS: Visual analog scale

\section{Introduction}

Temporomandibular disorders (TMD) are a subclass of musculoskeletal disorders resulting from dysfunction of the stomatognathic system and affect the masticatory muscles, temporomandibular joint, and orofacial structures [1]. TMD have been identified as the main cause of nondental pain in the orofacial region, with jaw and facial pain the most common symptoms [1]. The reported incidence of TMD in North America is 5\%-12\% [2,3]. Since the 1970s, a multifactorial etiology has been proposed for TMD, wherein pain and dysfunction result from biopsychosocial factors $[4,5]$. These etiological contributing factors include structural conditions, psychological morbidities, and behavioral problems such as parafunctional habits [6-9].

Nonfunctional continuous clenching during the awake state is considered a contributing factor to masticatory muscle pain, which is one of the symptoms of TMD [10,11]. Furthermore, Hirose et al. [12] reported large stresses in the posterior part of the disc and retrodiscal tissue, and the stress remained constant during continuous clenching based on the disc displacement model analyzed using finite-element analysis.

In most studies that employed a questionnaire [13-16], surveys of awake clenching were based on self-reports, and most patients were instructed by dentists to "take care to avoid clenching" and that "you should not be clenching." However, patients may be unaware of the magnitude of bite force that is equivalent to clenching; therefore, they may not be able to follow the advice of the dentist, which may adversely affect the clinical course. Potentially, the rates of selfreported clenching and TMD symptoms may differ because TMD patients interpret clenching as a large bite force. This study aimed to investigate the relationship between the magnitude of bite force that is interpreted as clenching (imagined clenching level; ICL) and TMD symptoms to evaluate the suitability of the term clenching for interpreting bite force during behavioral therapy and counseling for TMD patients. 
Citation: $\quad$ Nishiyama A, Otomo N, Tsukagoshi K, Tobe S, Kino K (2014) Magnitude of Bite Force that is Interpreted as Clenching in Patients with Temporomandibular Disorders: A Pilot Study. Dentistry S2: S2-004. doi:10.4172/2161-1122.S2-004

Page 2 of 4

\section{Subjects and Methods}

\section{Subjects}

A total 78 subjects were selected based on inclusion and exclusion criteria from patients with TMD who were treated at the Temporomandibular Joint Clinic at Tokyo Medical and Dental University between August and December, 2012. The inclusion criteria were as follows: age $>19$ years and a diagnosis of TMD based on the Research Diagnostic Criteria for Temporomandibular Disorders (RDC/TMD) [17]. The exclusion criteria were as follows: (1) presence of pain associated with acute inflammation of the stomatognathic system; (2) presence of chronic arthritis such as rheumatoid arthritis; (3) missing teeth and/or presence of a removable denture, with the presence of a fixed partial denture for $>1$ year; and (4) the use of regular medications such as analgesics, anti-anxiety drugs, antidepressants, and psychotropics. Three calibrated dentists trained in TMD performed the examinations and diagnosed TMD. All subjects provided written informed consent. The Institutional Review Board approved the study protocol (No.787).

\section{Questionnaire}

All patients completed a questionnaire that evaluated gender, age, pain intensity at rest and during maximum mouth opening, and chewing, ICL, and quality of life (QOL) associated with the TMD, which was assessed using the temporomandibular disorder-related limitation of daily functions (LDF-TMD) questionnaire (LDF-TMDQ) [13].

Pain intensity at rest and during maximum mouth opening and chewing was estimated using a visual analog scale (VAS) comprising a 100 -mm line with "no pain" on the extreme left and "intolerable pain" on the extreme right. The patients were instructed to rate their most severe TMD-related pain experienced at rest and during maximum mouth opening and chewing. For the rest and mouth opening pain, patients were asked the status of the examination at that time. For chewing pain, patients were asked the status of the most recent examination.

ICL was also estimated using a VAS (ICL-VAS) comprising a 100$\mathrm{mm}$ line with "no bite (0\%)" on the extreme left and "maximum bite force that cannot be exceeded (100\%)" on the extreme right.

The LDF-TMD questionnaire (LDF-TMDQ) was developed by Sugisaki et al. [18]. The questionnaire included the following question: "How much does your present jaw problem prevent or limit you from the following daily activities?: (1) opening your mouth while eating large morsels of food; (2) grinding thin morsels of food; (3) clenching your teeth; (4) brushing your back teeth; (5) yawning; (6) talking for a long period; (7) using your jaw for a long period during meals; (8) performing activities at home, school, and/or work; (9) falling asleep soon after going to bed; and (10) sleeping continuously at night." For each item, the subject chose any one of five levels on a numerical rating scale from "no problem at all" (0 points) to "extremely difficult" (4 points). The scores were summed to form the total score, which ranged from 0 to 40 points, and was designated as the LDF-TMD score.

\section{Statistical Analysis}

The questionnaires of two respondents were excluded from the statistical analysis due to missing data. Data from the remaining 76 participants were analyzed (16 males, 60 females; mean age $41.1 \pm 16.5$ years). A univariate analysis was performed using the Chi-square test, t-test, and one-way analysis of variance (ANOVA). Pearson correlation coefficients were calculated to analyze the correlation between ICL-VAS and other factors.

Factors that may have influenced ICL were estimated by multiple regression analysis (step-wise method) using ICL-VAS as the dependent variable and age, gender, VAS scores of pain intensity at rest and during maximum mouth opening and chewing, and LDFTMD score as independent variables. All statistical analyses were performed using SPSS version 21.0 software (IBM Japan).

\section{Results}

The sample size was calculated using a $G^{\star}$ Power of 3.1 .8 (Power $=0.997$, effect size $=0.5$, $\alpha$-error $=0.05)$. The mean (SD) ICL-VAS was $77.4( \pm 18.7) \mathrm{mm}$ (males, $78.6( \pm 19.9) \mathrm{mm}$; females, 73.1 ( \pm 12.9 $\mathrm{mm})$ ), with no significant difference between genders (Table 1).

\begin{tabular}{|l|l|l|}
\hline & & Total \\
\hline $\mathrm{N}$ & & 76 \\
\hline Mean age: $y r(\mathrm{SD})$ & & $41.1(16.5)$ \\
\hline Female: $\mathrm{n}(\%)$ & & $60(78.9)$ \\
\hline RDC/TMD: $\mathrm{n}(\%)$ & Group 1 & $5(6.6)$ \\
\hline & Group 2 & $56(73.7)$ \\
\hline & Group 3 & $15(19.7)$ \\
\hline Mean ICL: $\mathrm{mm}(\mathrm{SD})$ & & $77.4(18.7)$ \\
\hline
\end{tabular}

$T M D$, temporomandibular disorders; RDC/TMD, research diagnostic criteria for TMD; ICL, imagined clenching level; Group 1, myofascial pain; Group 2, disk displacement; Group 3, arthralgia, osteoarthritis, or osteoarthrosis

Table 1: Subject demographic characteristics and the TMD type

The mean ICL-VAS score was not significantly different among patients stratified by the pathological classification of TMD based on RDC/TMD: group 1, myofascial pain; group 2, disc displacement; group 3, arthralgia, osteoarthritis, or osteoarthrosis (Table 2).

\begin{tabular}{|l|l|l|}
\hline RDC/TMD & ICL VAS: $\mathrm{mm}(\mathrm{SD})$ & \\
\cline { 1 - 2 } Group 1 $(n=5)$ & $74.4(18.3)$ & \multirow{2}{*}{0.921} \\
\cline { 1 - 2 } Group 2 $(n=56)$ & $77.8(20.7)$ & \\
\cline { 1 - 2 } Group 3 $(n=15)$ & $76.9(9.2)$ & \\
\cline { 1 - 2 } Total & $77.4(18.7)$ & \\
\hline
\end{tabular}

ICL, imagined clenching level; TMD, temporomandibular disorders; RDC/TMD, Research Diagnostic Criteria for Temporomandibular Disorders; VAS, visual analog scale; SD, standard deviation; a, one-way analysis of variance (ANOVA); Group 1, myofacial pain; Group 2, disk displacement; Group 3, arthralgia, osteoarthritis, or osteoarthrosis; *Significance at $\mathrm{P}<0.05$

Table 2: ICL in 76 patients with TMD stratified by pathological classification based on RDC/TMD

ICL-VAS was significantly correlated with pain intensity during maximum mouth opening and chewing, and with the LDF-TMD 
scores (Table 3). However, multiple regression analysis identified only the LDF-TMD score as significant (Table 4).

\begin{tabular}{|l|l|l|}
\hline & $\begin{array}{l}\text { Correlation } \\
\text { coefficient }\end{array}$ & P-value* $^{*}$ \\
\hline Pain intensity at rest & 0.141 & 0.223 \\
\hline $\begin{array}{l}\text { Pain intensity during maximum mouth } \\
\text { opening }\end{array}$ & 0.320 & $0.005^{*}$ \\
\hline Pain intensity during chewing & 0.363 & $0.001^{*}$ \\
\hline LDF-TMD score & 0.367 & $0.001^{*}$ \\
\hline
\end{tabular}

TMD, temporomandibular disorders; LDF-TMD, TMD-related limitation of daily functions *Significance at $\mathrm{P}<0.05$

Table 3: Correlation between ICL and TMD-related outcomes

\begin{tabular}{|l|l|l|l|}
\hline & P-value $^{*}$ & $\begin{array}{l}\text { Non standardized } \\
\text { coefficient }\end{array}$ & $\begin{array}{l}\text { Standardized } \\
\text { coefficient }\end{array}$ \\
\hline LDF-TMD score & $0.001^{*}$ & 1.239 & 0.367 \\
\hline
\end{tabular}

TMD, temporomandibular disorders; LDF-TMD, TMD-related limitation of daily functions. *Significance at $\mathrm{P}<0.05$

Table 4: Results of multiple regression analysis

\section{Discussion}

The subjects in this study interpreted the magnitude of bite force during clenching as relatively large at approximately $70 \%$ of the maximum bite force.

The mean bite force during mastication from the first bite to swallowing is approximately $30 \mathrm{~N}$ [19]. Because the maximum clenching force is $300-500 \mathrm{~N}$ [20,21], the mean bite force during mastication is believed to be approximately $6 \%-10 \%$ of the maximum clenching force. In the TMD patients in our study, the ICL was considerably greater than the magnitude of force that was actually used during function and parafunction.

According to a study by Farella et al. [11], the mean maximum duration of intentional clenching at $40 \%$ MVC was approximately 1.4 $\mathrm{min}$, while that of intentional clenching at 7.5\% MVC was $157.2 \mathrm{~min}$. This finding showed that tooth-clenching with a low magnitude of force could be sustained for long periods compared to tooth-clenching with a moderate or higher magnitude of force. Several studies have shown that sustained static contractions of the masticatory muscles result in unbearable pain and fatigue that prevent patients from continuing contraction [22-24]. Farella et al. [11] also reported that prolonged tooth-clenching with a low magnitude of force induced delayed soreness in the jaw elevator muscles. In other words, continuous light clenching (such as tooth contact) affects TMD symptoms more than brief tight clenching.

It has been speculated that patients do not continue to clench with excessive force intentionally. Many patients continue to clench with a low magnitude of force because the force may be too small to be noticed. In 2006, Sato et al. [25] first described daytime light clenching as a tooth contacting habit (TCH). TCH was defined as a habitual behavior in which the upper and lower teeth are continuously brought together with minimal force in a nonfunctional situation, i.e., contact but not clenching. Sato et al. [25] reported that TCH occurred in
$52.4 \%$ of TMD patients with pain, and TCH patients with pain lasting greater than 4 months were less likely to experience improvement in the pain following the first visit. The activity of the masticatory muscles in the intercuspal position was shown to be higher than the muscle activity at rest [26]. Using a radiowave-activated wrist vibrator, Chen et al. [27] showed that non-functional tooth contact during the daytime is nearly 4 -fold higher in patients with myogenous pain than in healthy controls. Michelotti et al. [28] also reported that the habit of maintaining tooth contact was a significant risk factor for myofacial pain. Given that the average total time of functional tooth contact during chewing or swallowing is only $17.5 \mathrm{~min} /$ day [29], it is believed that continuous nonfunctional tooth contact causes overload of the TMJ and masticatory muscles. In fact, it has been shown that the association between clenching and muscle pain is due either to damaged muscle fibers or decreased blood supply to these fibers because the perfusion of the masseter was significantly lessened [30-35] during clenching. Therefore, TCH also affected the onset, persistence, and aggravation of TMD.

At our facility, behavior modification is used to control TCH. Behavior modification is defined as the use of empirically demonstrated techniques to improve behavior. Behavior modification targets behaviors that can be objectively measured and aims to control these behaviors by reinforcing adaptive behavior and reducing maladaptive behavior. This process includes behavioral assessment to measure the target behavior and subsequent modifications to change that behavior. We propose that $\mathrm{TCH}$ can be effectively controlled using a time sampling method of behavioral assessment and the habit reversal method for modification. This strategy includes three steps. The first step is a "motivation strategy," wherein the patient confirms the habitual behavior using reminders such as tags, stickers, and timers. The second step entails "awareness training" and "competing response training," wherein the patient performs a substitute action in place of the adverse habitual behavior (for example, taking a deep breath) immediately after noticing the behavior through a reminder. After performing these tasks, the patient experiences less jaw muscle strain. The final step is "reinforcement"; the patient increases the frequency of noticing the behavior by performing the first and second steps repeatedly. However, the efficacy of this technique has not been investigated.

In this study, because the subjects are TMD patients, it is impossible to determine whether the reported ICL is identical to that in the healthy population or other disease populations. Therefore, future studies are needed that evaluate multiple subpopulations.

\section{Conclusions}

TMD patients imagined the clenching force at a magnitude of approximately $70 \%$ of the maximum bite force. Patients are unlikely to be able to sustain clenching at this magnitude; therefore, clenching is not a term recommending for use during counseling and behavioral therapy of TMD patients.

\section{References}

1. Okeson JP (2005) Bell's orofacial pains: The clinical management of orofacial pain. (6thedn), Quintessence Publishing, Chicago.

2. Rugh JD, Solberg WK (1985) Oral health status in the United States: temporomandibular disorders. J Dent Educ 49: 398-406.

3. Dworkin SF, Huggins KH, LeResche L, Von Korff M, Howard J, et al. (1990) Epidemiology of signs and symptoms in temporomandibular 
Citation: Nishiyama A, Otomo N, Tsukagoshi K, Tobe S, Kino K (2014) Magnitude of Bite Force that is Interpreted as Clenching in Patients with Temporomandibular Disorders: A Pilot Study. Dentistry S2: S2-004. doi:10.4172/2161-1122.S2-004

Page 4 of 4

disorders: Clinical signs in cases and controls. J Am Dent Assoc 120: 273-281.

4. Weinberg LA (1974) Temporomandibular dysfunctional profile: a patient-oriented approach. J Prosthet Dent 32: 312-325.

5. Oral K, Bal Küçük B, Ebeoğlu B, Dinçer S (2009) Etiology of temporomandibular disorder pain. Agri 21: 89-94.

6. Moss RA, Garrett JC (1984) Temporomandibular joint dysfunction syndrome and myofacial pain dysfunction syndrome: a critical review. J Oral Rehabil 11: 3-28.

7. Schiffman EL, Fricton JR, Haley D (1992) The relationship of occlusion, parafunctional habits and recent life events to mandibular dysfunction in a non-patient population. J Oral Rehabil 19: 201-223.

8. Laskin DM (1969) Etiology of the pain-dysfunction syndrome. J Am Dent Assoc 79: 147-153.

9. Yap AU, Dworkin SF, Chua EK, List T, Tan KB, et al. (2003) Prevalence of temporomandibular disorders subtypes, psychologic distress, and psychosocial dysfunction in Asian patients. J Orofac Pain 17: 21-28.

10. Christensen LV (1979) Some subjective-experimental tooth clenching in man. J Oral Rehabil 6: 119-136.

11. Farella M, Soneda K, Vilmann A, Thomsen CE, Bakke M (2010) Jaw muscle soreness after tooth-clenching depends on force level. J Dent Res 89: 717-721.

12. Hirose M, Tanaka E, Tanaka M, Fujita R, Kuroda Y, et al. (2006) Threedimensional finite-element model of the human temporomandibular joint disc during prolonged clenching. Eur J Oral Sci 114: 441-448.

13. Minghelli B, Cardoso I, Porfírio M, Gonçalves R, Cascalheiro S, et al. (2014) Prevalence of temporomandibular disorder in children and adolescents from public schools in Southern Portugal. N Am J Med Sci 6: 126-132.

14. Paesani DA, Lobbezoo F, Gelos C, Guarda-Nardini L, Ahlberg J, Manfredini D (2013) Correlation between self-reported and clinically based diagnoses of bruxism in temporomandibular disorders patients. J Oral Rehabil 40: 803-809.

15. Bagis B, Ayaz EA, Turgut S, Durkan R, Özcan M (2012) Gender difference in prevalence of signs and symptoms of temporomandibular joint disorders: a retrospective study on 243 consecutive patients. Int $J$ Med Sci 9: 539-544.

16. Mobilio N, Casetta I, Cesnik E, Catapano S (2011) Prevalence of selfreported symptoms related to temporomandibular disorders in an Italian population. J Oral Rehabil 38: 884-890.

17. Dworkin SF, LeResche L (1992) Research diagnostic criteria for temporomandibular disorders: review, criteria, examinations and specifications, critique. J Craniomandib Disord 6: 301-355.

18. Sugisaki M, Kuruma R, Kino K, Shibuya T, Tsukahara H, et al. (2007) Selection of question items for screening patients with temporomandibular disorders and estimation of their validity. J Jpn Soc TMJ 19: 177-184.

19. De Boever JA, McCall WD, Holden S, Ash MM Jr (1978) Functional occlusal forces: an investigation by telemetry. J Prosthet Dent 40: 326-333.
20. Meena A, Jain V, Singh N, Arora N, Jha R (2013) Effect of implantsupported prosthesis on the bite force and masticatory efficiency in subjects with shortened dental arches. J Oral Rehabil 41: 87-92.

21. Gonçalves TM, Campos CH, Gonçalves GM, de Moraes M, Rodrigues Garcia RC (2013) Mastication improvement after partial implantsupported prosthesis use. J Dent Res 92: 189S-194S.

22. Clark GT, Carter MC (1985) Electromyographic study of human jawclosing muscle endurance, fatigue, and recovery at various isometric force levels. Arch Oral Biol 30: 563-569.

23. Clark GT, Jow RW, Lee JJ (1989) Jaw pain and stiffness levels after repeated maximum voluntary clenching. J Dent Res 68: 69-71.

24. Christensen LV (1989) Experimental teeth clenching in man. Swed Dent J Suppl 60: 1-66.

25. Sato F, Kino K, Sugisaki M, Haketa T, Amemori Y, et al. (2006) Teeth contacting habit as a contributing factor to chronic pain in patients with temporomandibular disorders. J Med Dent Sci 53: 103-109.

26. Michelotti A, Farella M, Vollaro S, Martina R (1997) Mandibular rest position and electrical activity of the masticatory muscles. J Prosthet Dent 78: 48-53.

27. Chen CY, Palla S, Erni S, Sieber M, Gallo LM (2007) Nonfunctional tooth contact in healthy controls and patients with myogenous facial pain. J Orofac Pain 21: 185-193.

28. Michelotti A, Cioffi I, Festa P, Scala G, Farella M (2010) Oral parafunctions as risk factors for diagnostic TMD subgroups. J Oral Rehabil 37: 157-162.

29. Graf H (1969) Bruxism. Dent Clin North Am 13: 659-665.

30. Buzinelli RV, Berzin F (2001) Electromyographic analysis of fatigue in temporalis and masseter muscles during continuous chewing. J Oral Rehabil 28: 1165-1167.

31. Svensson P, Burgaard A, Schlosser S (2001) Fatigue and pain in human jaw muscles during a sustained, low-intensity clenching task. Arch Oral Biol 46: 773-777.

32. Kim YJ, Kuboki T, Tsukiyama Y, Koyano K, Clark GT (1999) Haemodynamic changes in human masseter and temporalis muscles induced by different levels of isometric contraction. Arch Oral Biol 44: 641-650.

33. Larsson SE, Bengtsson A, Bodegard L, Henriksson KG, Larsson J (1988) Muscle changes in work-related chronic myalgia. Acta Orthop Scand 59: 552-556.

34. Larsson B, Bjork J, Kadi F, Lindman R, Gerdle B (2004) Blood supply and oxidative metabolism in muscle biopsies of female cleaners with and without myalgia. Clin J Pain 20: 440-446.

35. Nakamura Y, Torisu T, Noguchi K, Fujii H (2005) Changes in masseter muscle blood flow during voluntary isometric contraction in humans. J Oral Rehabil 35: 545-551.
This article was originally published in a special issue, entitled: "Chornic Orofacial Pain or Temporomandibular Disorders", Edited by Dr. Francisco Alencar Jr., Marquette University, USA 